\title{
Algunas características asociadas con la presencia de indicadores de Burnout en profesionales de salud mental panameños
}

\section{Rubén Díaz H.,"}

${ }^{1}$ Profesor, Escuela de Psicología, Universidad Santa María la Antigua, Apartado Postal 0819-08550, Panamá, República de Panamá.

*Autor para correspondencia. Email: rdiazh@usma.com.pa

Recibido: 7 de marzo de 2014

Aceptado: 15 de abril de 2014

\begin{abstract}
Burnout syndrome (BS) is a response towards chronic work stress in which the person develops feelings of emotional exhaustion, uses depersonalization as a defense, and adopt attitudes of diminished sense of professional accomplishment. It develops as a result of ineffective coping strategies toward work stress. This study tries to evaluate BS prevalence and its relation to coping strategies and role stressors, in a sample of 92 mental health professionals who work in hospitals at City of Panama. Four self-report scales (General data survey, MBI, Brief COPE, Role conflict and ambiguity scale) were used to assess variables of interest. A prevalence of $27.2 \%$ participants with some level of BS was found. Emotional exhaustion dimension was the most frequent in the sample. Important relations are found with years of service, role ambiguity, and dysfunctional- and emotion-centered coping strategies.
\end{abstract}

Keywords: Burnout, Mental health professional, Coping strategies, Role stressors, Emotional exhaustion.

\section{Resumen}

El Síndrome de Burnout (SB) es una respuesta al estrés laboral crónico que involucra el desarrollo de sentimientos de agotamiento emocional, utilización de despersonalización como defensa y actitud de baja realización personal, y que surge cuando las estrategias de afrontamiento empleadas resultan ineficaces para enfrentar dicho estrés. Esta investigación evalúa la prevalencia del SB y su relación con estrategias de afrontamiento y estresores de rol, en una muestra de 92 profesionales de salud mental que laboran en hospitales en la Ciudad de Panamá. Se utilizan 
Invest. pens. crit.

Vol. 2, No. 3, enero-abril 2014.

pp. $13-25$

cuatro escalas de auto-reporte (Cuestionario de datos generales, MBI, COPE breve y Escala de conflicto y ambigüedad de rol), para recopilar los datos. Se encuentra una prevalencia de $27.2 \%$ participantes con algún nivel de SB, siendo la dimensión de agotamiento emocional la de más frecuente aparición. Se hallan correlaciones importantes con los años de servicio en la institución, ambigüedad de rol y con estrategias de afrontamiento disfuncionales y centradas en la emoción.

Palabras clave: Desgaste profesional, Profesionales de salud mental, Afrontamiento, Estresores de rol, Agotamiento emocional.

\section{Introducción}

Algunas características asociadas con la presencia de Síndrome de Burnout en profesionales de salud mental panameños: Estrategias de afrontamiento y estresores de rol. El Síndrome de Burnout (SB) es un problema que tiene implicaciones graves desde el punto de vista del individuo que lo padece y de las organizaciones en donde está inserto (Gascón, Olmedo Montes y Ciccotelli, 2003). El SB puede definirse como respuesta al estrés laboral crónico a nivel personal y relacional, que surge cuando las estrategias de afrontamiento empleadas resultan ineficaces para enfrentar dicho estrés, y que consiste en el desarrollo de sentimientos de agotamiento emocional, la utilización de la despersonalización como estrategia de afrontamiento y en actitudes de baja realización personal en el trabajo (Gil-Monte y Peiró, 1997; Maslach y Jackson, 1984).

El SB tiene consecuencias a nivel individual tanto físicas como psicológicas, entre las que se destacan sensación de deterioro personal, incremento en uso de drogas, aumento de agresividad, dificultades respiratorias y cardiovasculares, deterioro familiar y aislamiento (Borritz, 2006; Fidalgo, 2007; Grau, Suñer y García, 2005; Martínez, 2010; Melamed, Shirom, Toker, Berliner y Shapira, 2006; Olmedo, Santed, Jiménez y Gómez, 2001; Quass, 2008; Shirom, Nirel y Vinokur, 2006). Por otra parte, puede generar consecuencias a nivel de la eficiencia de una organización para el logro de sus objetivos ya que puede producir desmejoras en la calidad del servicio que brinda el profesional que lo padece y también en el que brinda su equipo de trabajo. Estudios (Bakker, Le Blanc y Schaufeli, 2005; Bakker, Westman y Schaufeli, 2007; Bakker y Schaufeli, 2000) han encontrado evidencia de lo que denominan "contagio" del SB: esto es que la presencia de un colaborador con SB puede generar SB en el resto del equipo de trabajo de manera directa o indirecta.

En el caso de los profesionales de salud mental las características laborales de contacto directo y continuo con el cliente y sus patologías pueden aumentar el riesgo a desarrollar SB (Bosqued, 2008; Olmedo y cols., 2001; Zur, 2007). El contacto frecuente con la enfermedad mental enfrenta al profesional de la salud con temores asociados a su propia vulnerabilidad (Buunk y Schaufeli, 1993; Miller, 2005). 
Invest. pens. crit.

Vol. 2, No. 3, enero-abril 2014.

pp. $13-25$

Las relaciones que establecen los profesionales de salud mental con sus pacientes suelen ser caracterizadas por una falta de equilibrio en los intercambios sociales con sus pacientes (Buunk y Schaufeli, 1993). Además, puede presentarse un desequilibrio entre las demandas del trabajo y los recursos con los que se cuenta para llevarlas a cabo (Lorente, Salanova, Martínez y Schaufeli, 2008).

En Panamá se ha estudiado el SB en poblaciones de maestros, médicos y enfermeras (Bosquez, 2001; Castillo, 2007; Delvalle, 2006; Grimaldo, 2005; Matteus, 2004; Ortega, Salas y Correa, 2011; Rodes, 2006), estimando la prevalencia en estas poblaciones de entre el 4 y el 18\% sujetos.

El presente artículo deriva de una investigación desarrollada en el año 2010 (Díaz y Rodríguez, 2010) para estimar la prevalencia del síndrome de burnout en una muestra de psiquiatras, psicólogos clínicos y enfermeras especialistas en salud mental que laboran en instituciones de salud en el sistema público. Tiene como objetivo evaluar algunas características personales y organizacionales en relación con la presencia del síndrome de burnout en profesionales de salud mental de cuatro hospitales de la Ciudad de Panamá. La elección de las características personales y organizacionales a ser consideradas en este artículo se basa en los modelos propuestos por GilMonte y Peiró (1997) y por Gil-Monte, Peiró y Valcárcel (1998) según su función como Desencadenantes y Facilitadores.

\section{Material y métodos}

\section{$\underline{\text { Participantes }}$}

Participaron voluntariamente en el estudio noventa y dos profesionales de salud mental del sector público de salud -19 médicos psiquiatras, 59 enfermeras especialistas en salud mental y 15 psicólogos clínicos - con edades entre 25 y 67 años $(M d n=47 ; M A D=6.7)$, y tiempo mediano de servicio en la institución de 17 años $(M A D=10.3)$.

La cantidad de sujetos que participaron del estudio corresponde al 74\% del total de profesionales de salud mental que laboraban, en el momento del estudio, en los cuatro hospitales seleccionados de manera intencional: Hospital del Niño, Hospital Santo Tomás, Instituto Nacional de Salud Mental y Complejo Hospitalario Arnulfo Arias Madrid.

\section{$\underline{\text { Medidas }}$}

Se utilizaron cuatro cuestionarios de auto-reporte para obtener información sobre las variables de interés: Síndrome de Burnout, variables desencadenantes (estresores de rol y condición laboral) y variables facilitadoras (estrategias de afrontamiento).

Cuestionario de datos generales. Diseñado específicamente para los fines de este estudio, contiene preguntas acerca de la condición personal y profesional del entrevistado. La caracterización 
Invest. pens. crit.

Vol. 2, No. 3, enero-abril 2014.

pp. $13-25$

personal incluía preguntas sociodemográficas de sexo y edad. Para indagar sobre la condición laboral de los participantes, además de preguntar sobre su profesión, se incluían preguntas sobre los años de servicio en la institución actual, si ha trabajado anteriormente en otra institución pública de salud y si trabaja simultáneamente en consulta privada.

Inventario "Burnout" de Maslach (MBI). Es la adaptación al español por Nicolás Seisdedos del inventario creado por Christina Maslach y Susan Jackson (1997). Contiene 22 preguntas con escala de respuesta tipo Likert de 7 puntos, relativos a la frecuencia en que se experimentan las situaciones descritas. Permite la evaluación de tres dimensiones de Burnout: Agotamiento emocional (AE) se refiere a una progresiva pérdida de energía y un creciente desgaste de los recursos emocionales; la despersonalización (DP) que se refiere a actitudes de distanciamiento frente a los problemas y personas con que trata que surge como una defensa que permite establecer una barrera emocional para ignorar las cualidades que hacen únicas a éstas; y la realización profesional en el trabajo (RP) que tiene que ver con la percepción y sentimiento de competencia profesional (Bosqued, 2008; Maslach y Jackson, 1984; Maslach, Leiter y Schaufeli, 2008).Las puntuaciones para cada escala se obtienen sumando los valores de los ítems respectivos. Para este estudio se utilizarán los puntos de corte para la adaptación española por Seisdedos (Maslach y cols., 1997), con el fin de determinar el grado de la dimensión: alta, media o baja.

Se calculó el coeficiente alfa de Cronbach y se encontró que para los datos de esta investigación las escalas de $\mathrm{AE}(\alpha=.83 ; S E M=3.8)$ y de RP $(\alpha=.73 ; S E M=3.5)$ presentan una consistencia interna inter-ítem adecuada, mientras que los resultados de la escala de DP $(\alpha=$ .32; $S E M=2.2$ ) indican cautela en su interpretación.

Para evaluar la presencia de SB se clasificó el nivel de Burnout presentado por los sujetos de acuerdo siguiendo el método utilizado por Rubio (2003). Para los efectos de este artículo, los niveles poco, medio, bastante y extremo corresponderán a presencia de SB, mientras que el nivel nada indicará ausencia de SB.

Escala Breve de Evaluación de Técnicas de Afrontamiento (COPE Breve) modificado. Es una escala que tiene como objetivo evaluar las estrategias de afrontamiento en sus modos cognitivo y conductual (Perczek, Carver, Price y Pozo-Kaderman, 2000). La versión utilizada en este estudio toma los 24 reactivos de la escala de Perczek y cols. (2000) y añade 4 reactivos de tomados de la traducción del instrumento original por Crespo y López (2003) para incluir las dos estrategias de afrontamiento - búsqueda de apoyo instrumental y autoculparse- considerando su relevancia según estudios previos (Quass, 2008; Shimizutani y cols., 2008; Udipi, Veach, Kao y LeRoy, 2008). Los 28 reactivos permiten evaluar 14 estrategias de afrontamiento que pueden 
Invest. pens. crit.

Vol. 2, No. 3, enero-abril 2014.

pp. $13-25$

agruparse en tres categorías: centradas en el problema, centradas en la emoción y disfuncionales (Carver, Scheier yy Weintraub, 1989).

Escala de conflicto y ambigüedad de rol. La escala es una traducción (Arquer, Daza yy Nogareda, 1995) del cuestionario elaborado por Rizzo, House y Lirtzman en 1980, que consta de 13 reactivos para medir dos estresores de rol: ambigüedad de rol y conflicto de rol. Los reactivos presentan una escala de diferencial semántico de 7 puntos que debe ser completada por el examinado según su percepción de la magnitud con que el reactivo describe su situación laboral. El análisis de confiabilidad revela una consistencia interna inter-ítem adecuada para ambas subescalas de conflicto $(\alpha=.82 ; S E M=5.1)$ y ambigüedad de rol $(\alpha=.66 ; S E M=2.2)$.

\section{$\underline{\text { Procedimiento }}$}

Una vez obtenido el consentimiento informado de todos los participantes, se les aplicaron tres cuestionarios de auto-reporte de manera individual para ser contestados de manera anónima. El orden de presentación de los cuestionarios fue el siguiente: Cuestionario de datos generales, MBI, COPE breve y Escala de conflicto y ambigüedad de rol.

\section{Resultados}

Se inicia el análisis de resultados con una descripción de la prevalencia del SB y de cada uno de sus componentes en la muestra estudiada. Más adelante, se evalúa la intensidad de la relación entre las distintas variables analizadas en la investigación, con especial énfasis en la relación con las variables de $\mathrm{SB}$.

\section{Prevalencia y caracterización del SB}

La Figura 1 presenta la distribución de los participantes según el nivel de SB que presentan. Se observa que 35 individuos de un total de 92 participantes presentan algún nivel de SB, lo que representa una prevalencia del $27.2 \%$. Es destacable que la mayoría de estos sujetos presenta un nivel bajo de SB y solamente 1 presenta un nivel extremo, que corresponde con la definición original de SB según Maslach y Jackson (1984). 
Invest. pens. crit.

Vol. 2, No. 3, enero-abril 2014.

pp. $13-25$

Figura 1. Distribución de participantes según nivel de SB.

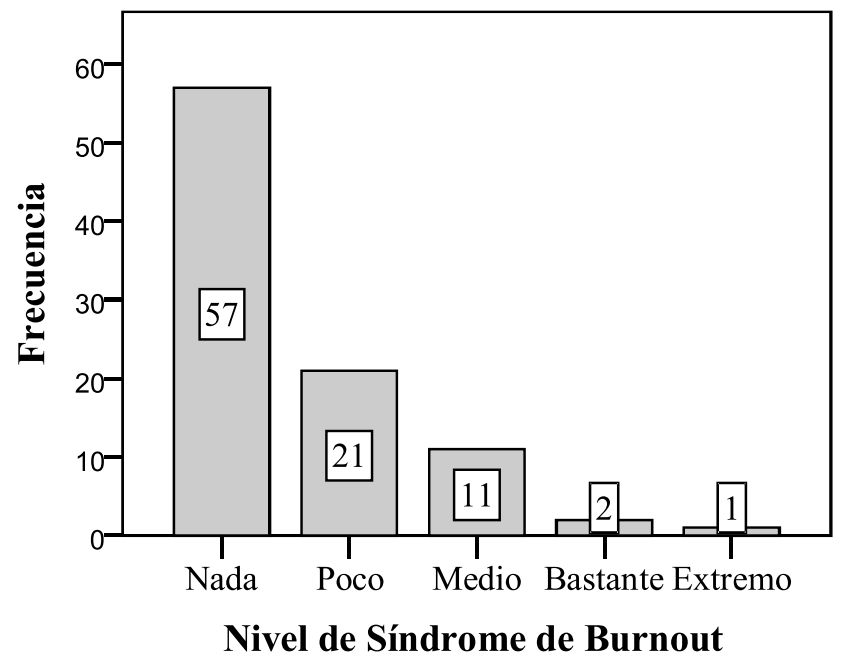

Cuando se estudia cada una de las dimensiones de SB por separado, se encuentra que hay una mayor presencia de agotamiento emocional en la muestra estudiada, que en sus grados medio y alto agrupa al 50\% de sujetos. Como se observa en la Tabla 1, en las otras dos dimensiones la mayor parte de los participantes no están incluidos en los grados indicadores de SB.

Tabla 1

Distribución de 92 participantes según el grado de cada una de las dimensiones de Burnout

\begin{tabular}{llll}
\hline Dimensión de Burnout & Bajo (\%) & Medio (\%) & Alto (\%) \\
Agotamiento emocional & 48.9 & 34.8 & 16.3 \\
Despersonalización & 82.6 & 16.3 & 1.1 \\
Realización personal & 8.7 & 20.7 & 70.7 \\
\hline
\end{tabular}

Los 35 participantes que presentan SB en algún nivel tienen edades entre 25 y 58 años $(M d n=$ 45 ; $M A D=5.2)$, tiempo mediano de servicio en la institución de 16 años $(M A D=7.4)$, características similares al grupo de participantes que no presentan SB.

\section{$\underline{\text { Matriz de correlaciones }}$}

La Tabla 2 muestra los coeficientes de correlación entre las variables de estudio. En vista de que la mayoría de las variables no se distribuye normalmente se utilizan la mediana $(M d n)$ y la desviación absoluta de la mediana $(M A D)$ como medidas de tendencia central y dispersión, y el coeficiente rho de Spearman como coeficiente de correlación.

Del grupo de variables personales y de historia laboral, solamente la variable de años de servicio en la instalación actual presenta correlación negativa de magnitud moderada con la dimensión de agotamiento emocional. 
Invest. pens. crit.

Vol. 2, No. 3, enero-abril 2014.

pp. $13-25$

En el caso de las estrategias de afrontamiento, se observa que hay mayor cantidad de estrategias disfuncionales que tienen correlaciones con magnitudes, por lo menos, moderadas con las dimensiones del SB. Destacan la negación, la desconexión conductual y el autoculparse como estrategias relacionadas con la dimensión de despersonalización; y las estrategias de autoculparse y uso de drogas con la dimensión de agotamiento emocional. Por otra parte, el uso de la religión como estrategia de afrontamiento centrado en la emoción se correlaciona negativamente con el agotamiento emocional.

Con respecto a los estresores de rol, se observa que la ambigüedad de rol se correlaciona positivamente con la estrategia de despersonalización. 
Invest. pens. crit.

Vol. 2, No. 3, enero-abril 2014.

pp. 13-25

\begin{tabular}{|c|c|c|c|c|c|c|c|c|c|c|c|c|c|c|c|c|c|c|c|c|}
\hline & $\underline{1}$ & $\underline{2}$ & $\underline{3}$ & $\underline{4}$ & $\underline{5}$ & $\underline{6}$ & $\underline{7}$ & $\underline{8}$ & $\underline{9}$ & $\underline{10}$ & $\underline{11}$ & $\underline{12}$ & $\underline{13}$ & $\underline{14}$ & $\underline{15}$ & $\underline{16}$ & $\underline{17}$ & $\underline{18}$ & $\underline{19}$ & $\underline{20}$ \\
\hline \multicolumn{21}{|l|}{ Dimensiones de SB } \\
\hline 1. Agotamiento emocional & --- & & & & & & & & & & & & & & & & & & & \\
\hline 2. Despersonalización & .12 & --- & & & & & & & & & & & & & & & & & & \\
\hline 3. Realización personal & .39 & -.04 & --- & & & & & & & & & & & & & & & & & \\
\hline \multicolumn{21}{|l|}{ Variables personales y de historia laboral } \\
\hline 4. Edad & -.11 & -.22 & .07 & --- & & & & & & & & & & & & & & & & \\
\hline 5. Años de servicio en instalación actual & -.42 & -.18 & -.11 & $.72^{* *}$ & --- & & & & & & & & & & & & & & & \\
\hline \multicolumn{21}{|c|}{ Estrategias de afrontamiento centradas en el problema } \\
\hline 6. Afrontamiento activo & .12 & .02 & -.08 & .06 & .25 & --- & & & & & & & & & & & & & & \\
\hline 7. Búsqueda de apoyo instrumental & .07 & -.08 & -.02 & .37 & .27 & .39 & --- & & & & & & & & & & & & & \\
\hline 8. Planificación & .06 & -.01 & .24 & .14 & .49 & $.80^{* *}$ & .34 & --- & & & & & & & & & & & & \\
\hline \multicolumn{21}{|c|}{ Estrategias de afrontamiento centradas en la emoción } \\
\hline 9. Aceptación & .24 & -.17 & .27 & .31 & .41 & $.80^{* *}$ & .46 & $.79^{* *}$ & --- & & & & & & & & & & & \\
\hline 10. Búsqueda de apoyo emocional & .23 & -.20 & .12 & .29 & .40 & $.88^{* *}$ & $.56^{*}$ & $.86^{* *}$ & $.92^{* *}$ & --- & & & & & & & & & & \\
\hline 11. Humor & -.20 & .21 & .02 & .36 & $.54^{*}$ & .26 & .19 & .50 & .12 & .34 & --- & & & & & & & & & \\
\hline 12. Reinterpretación positiva & .21 & .35 & .31 & -.06 & .30 & .45 & -.08 & $.72^{* *}$ & .41 & .42 & .47 & --- & & & & & & & & \\
\hline 13. Religión & -.42 & -.03 & -.22 & $.54^{*}$ & $.70^{* *}$ & .35 & .49 & .30 & .33 & .39 & $.59^{*}$ & .14 & --- & & & & & & & \\
\hline \multicolumn{21}{|c|}{ Estrategias de afrontamiento disfuncionales } \\
\hline 14. Autoculparse & $.56^{*}$ & .40 & .10 & -.25 & -.35 & .22 & .27 & .14 & .25 & .25 & -.25 & .23 & -.31 & --- & & & & & & \\
\hline 15. Autodistraerse & -.28 & .11 & .13 & .37 & $.64^{*}$ & .42 & $.60^{*}$ & $.66^{*}$ & $.58^{*}$ & $.56^{*}$ & .49 & .42 & $.61^{*}$ & .11 & --- & & & & & \\
\hline 16. Desahogo & .39 & -.01 & .15 & .06 & .16 & $.80^{* *}$ & $.56^{*}$ & $.65^{*}$ & $.88^{* *}$ & $.83^{* *}$ & .01 & .39 & .34 & .36 & .46 & --- & & & & \\
\hline 17. Desconexión conductual & -.13 & .51 & .07 & .24 & .29 & .12 & -.09 & .32 & .10 & .11 & .40 & $.57^{*}$ & .09 & .36 & .41 & -.08 & --- & & & \\
\hline 18. Negación & .30 & $.72^{* *}$ & .06 & -.03 & -.10 & .22 & .37 & .14 & .04 & .08 & .13 & .43 & .12 & $.584^{*}$ & .25 & .29 & .46 & --- & & \\
\hline 19. Uso de drogas & $.62^{*}$ & .03 & .17 & .07 & .01 & .45 & $.62^{*}$ & .41 & $.56^{*}$ & $.56^{*}$ & -.04 & .34 & .12 & .40 & .29 & $.80^{* *}$ & -.12 & .47 & --- & \\
\hline 20. Conflicto de rol & .06 & .34 & .14 & -.32 & -.18 & .32 & .11 & .36 & .07 & .22 & .31 & .35 & -.02 & .24 & -.01 & .18 & .24 & .35 & .14 & --- \\
\hline 21. Ambigüedad de rol & -.06 & $.67^{* *}$ & .10 & -.48 & -.53 & -.11 & -.08 & -.15 & -.38 & -.38 & -.08 & .01 & -.36 & .13 & -.09 & -.21 & .14 & .48 & -.09 & .36 \\
\hline
\end{tabular}

$* \mathrm{p}<.05 ; * * \mathrm{p}<.01$ 
Invest. pens. crit.

Vol. 2, No. 3, enero-abril 2014.

pp. $13-25$

\section{Discusión}

La presente investigación fue diseñada con dos objetivos principales: primero, estimar la prevalencia de SB en una muestra de profesionales de salud mental y, segundo, explorar las relaciones del SB con variables personales, de historia laboral, estresores de rol y estrategias de afrontamiento.

Para el primer objetivo, los resultados obtenidos evidencian una prevalencia de $27.2 \%$ participantes con algún grado de SB. Este resultado presenta una diferencia importante con estudios previos en Panamá (Bosquez, 2001; Delvalle, 2006; Ortega y cols., 2011) utilizando poblaciones de profesionales de salud. Es importante notar que si bien en los estudios reportados se utilizó la misma escala que en la presente investigación (MBI), difieren en el procedimiento para identificar el SB: algunas indican presencia de SB si se presentan niveles altos en por lo menos una dimensión, mientras que otras restringen la categoría solamente a aquellos participantes que muestran alto agotamiento emocional, alta despersonalización y baja realización personal. En ese sentido, es requerida una estandarización en la definición del SB para su adecuada identificación (Schaufeli, Leiter y Maslach, 2009).

En el presente estudio, la dimensión de agotamiento emocional apareció en grado indicador de SB con mayor frecuencia que las otras dos dimensiones. Esto concuerda con lo encontrado por Bosquez (2001) pero difiere de lo reportado por Grimaldo (2005), Castillo (2007) y Ortega y cols. (2011) quienes hallan la baja realización personal como la dimensión de mayor frecuencia en sus respectivas muestras.

Cuando se evalúa la confiabilidad de la escala MBI, se encuentra en este estudio que las sub-escalas de agotamiento emocional y realización personal tienen coeficientes de consistencia interna inter-ítem adecuados, lo que no sucede con la sub-escala de despersonalización. Si bien la prueba ha sido adaptada y validada para poblaciones hispanoparlantes (García, Herrero y León, 2007; Manso, 2006; Maslach y cols., 1997), estos resultados aportan evidencia en la necesidad de considerar la vigencia del MBI como la prueba estándar para este síndrome (Alvarado, 2009; Maslach y cols., 2008; Schaufeli y cols., 2009).

En cuanto al segundo objetivo, se encuentran correlaciones importantes con la ambigüedad de rol lo que suma evidencia a lo reportado en estudios previos (Garrosa, Moreno, Rodríguez y Sanz, 2007; López, Osca y Rodríguez, 2008; Osca, González-Camino, Bardera y Peiró, 2003; Rubio, 2003).

Los resultados de la relación negativa con los años de servicio en la institución concuerdan con estudios previos (Grau y cols., 2005). En contraste, otros investigadores (Ortega y cols., 2011; PeredaTorales, Celedonio, Guillermo, Hoyos Vásquez y Yánez Zamora, 2009) encontraron una relación positiva con los años de servicio. Es necesario profundizar en la explicación de esta diferencia, que podría deberse a las características propias de las poblaciones estudiadas. En nuestro caso, el tiempo de servicio mediano fue de 17 años, similar al reportado por Pereda-Torales y cols.; en el estudio de Ortega y cols. la mitad de los sujetos tenían entre 1 y 10 años de servicio; y en el de Grau y cols. el tiempo promedio de servicio fue de 13 años. A diferencia de Rodes (2006) no encontramos evidencia para sugerir relación del SB con la edad. 
Invest. pens. crit.

Vol. 2, No. 3, enero-abril 2014.

pp. $13-25$

Las relaciones encontradas con las estrategias de afrontamiento disfuncionales (autoculparse, desconexión conductual, negación, uso de drogas) y con aquellas centradas en la emoción (religión) concuerdan con los resultados de investigaciones previas (Guerrero, 2003; Jenaro, Flores y Gonzalez, 2007; Quass, 2008; Shimizutani y cols., 2008). Sin embargo, no se hallan relaciones con las estrategias de afrontamiento centradas en el problema, que otros estudios han sido encontrados vínculos negativos entre estos y la presencia de SB (Guerrero, 2003; Jenaro y cols., 2007).

Es necesario continuar estudiando este fenómeno incluyéndolo como parte de un proceso organizacional y ajustándose a las nuevas tendencias que lo identifican como un extremo del continuum de engagement (implicación) con el trabajo.

\section{Referencias}

Alvarado, K. (2009). Validez factorial de Maslach Burnout Inventory (versión castellana) en educadores costarricenses. Actualidades Investigativas en Educación, 9(1), 1-22.

Arquer, M. I., Daza, F. M. y Nogareda, C. (1995). Ambigüedad y conflicto de rol (Nota técnica de prevención No. 388). España: Instituto Nacional de Seguridad e Higiene en el Trabajo.

Bakker, A. B., Le Blanc, P. M. y Schaufeli, W. B. (2005). Burnout contagion among intensive care nurses. Journal of advanced nursing, 51(3), 276-287.

Bakker, A. B. y Schaufeli, W. B. (2000). Burnout contagion processes among teachers. Journal of Applied Social Psychology, 30(11), 2289-2308.

Bakker, A. B., Westman, M. y Schaufeli, W. B. (2007). Crossover of burnout: An experimental design. European Journal of Work and Organizational Psychology, 16(2), 220-239. doi:10.1080/13594320701218288

Borritz, M. (2006). Burnout in human service work-causes and consequences: Results of 3 -years of follow-up of the puma study among human service workers in Denmark (Tesis doctoral). National Institute of Occupational Health, Dinamarca.

Bosqued, M. (2008). Quemados: el síndrome de burnout : qué es y cómo superarlo. Barcelona: Paidós [lbérica].

Bosquez, E. (2001). Estudio descriptivo sobre el síndrome de burnout en enfermeras y médicos hematólogos del C.H.M.DR.A.AM. y el I.O.N (Tesis para grado de licenciatura). Universidad de Panamá, Panamá.

Buunk, B. P. y Schaufeli, W. B. (1993). Burnout: A perspective from social comparison theory. Recuperado a partir de http://psycnet.apa.org/psycinfo/1993-97794-004

Carver, C. S., Scheier, M. F. y Weintraub, J. K. (1989). Assessing coping strategies: A theoretically based approach. Journal of Personality and Social Psychology, 56(2), 267-283. 
Invest. pens. crit.

Vol. 2, No. 3, enero-abril 2014.

pp. $13-25$

Castillo, A. (2007). Estudio descriptivo sobre el síndrome de burnout (Tesis para grado de licenciatura). Universidad de Panamá, Panamá.

Crespo, M. y López, L. (2003). COPE Breve. En M. Crespo y F. J. Labrador (Eds.), Estrés. Madrid: Editorial Síntesis.

Delvalle, B. (2006). Estudio de ansiedad y depresión en personal profesional de la salud, Hospital Rafael Estévez (Tesis para grado de maestría). Universidad de Panamá, Panamá.

Díaz, R. y Rodríguez, C. (2010). Estudio sobre el síndrome de desgaste profesional (Burnout) en profesionales de salud mental que laboran en las unidades de la Caja de Seguro Social y del Ministerio de Salud en el área metropolitana de la ciudad de Panamá. (Tesis para grado de licenciatura). Universidad Católica Santa María la Antigua, Panamá.

Fidalgo, V. (2007). Síndrome de estar quemado por el trabajo o burnout(II): consecuencias, evaluación y prevención (Nota técnica de prevención No. 705). España: Instituto Nacional de Seguridad e Higiene en el Trabajo.

García, J., Herrero, S. y León, J. L. (2007). Validez factorial del Maslach Burnout Inventory (MBI) en una muestra de trabajadores del Hospital Psiquiátrico Penitenciario de Sevilla. Apuntes de Psicología, 25(2), 157-174.

Garrosa, E., Moreno, B., Rodríguez, A. y Sanz, A. (2007). La influencia del estrés de rol y la competencia emocional en el desgaste profesional y en el engagement. Información Psicológica, (91/92), 80-94.

Gascón, S., Olmedo Montes, M. y Ciccotelli, H. (2003). La prevención del burnout en las organizaciones: el chequeo preventivo de Leiter y Maslach. Revista de psicopatología y psicología clínica, 8(1). Recuperado a partir de http://e-spacio.uned.es/revistasuned/index.php/RPPC/article/view/3942

Gil-Monte, P. y Peiró, J. (1997). Desgaste psíquico en el trabajo: el síndrome de quemarse. Madrid: Sintesis.

Gil-Monte, P., Peiró, J. y Valcárcel, P. (1998). A model of burnout process development: An alternative from appraisal models of stress. Comportamento Organizacional e gestâo, 2(2), 211-220.

Grau, A., Suñer, R. y García, M. M. (2005). Desgaste profesional en el personal sanitario y su relación con los factores personales y ambientales. Gaceta sanitaria, 19(6), 463-470.

Grimaldo, G. (2005). Factores psicológicos y sus efectos en el desempeño laboral de los docentes del programa de postgrado del nivel superior de la Facultad de Ciencias de la Educación de la Universidad de Panamá (Tesis para grado de maestría). Universidad de Panamá, Panamá.

Guerrero, E. (2003). Análisis pormenorizado de los grados de burnout y técnicas de afrontamiento del estrés docente en profesorado universitario. Anales de Psicología, 19(1), 145-158. 
Invest. pens. crit.

Vol. 2, No. 3, enero-abril 2014.

pp. $13-25$

Jenaro, C., Flores, N. y González, F. (2007). Síndrome de burnout y afrontamiento en trabajadores de acogimiento residencial de menores. International Journal of Clinical and Health Psychology, 17, 107-121.

López, B., Osca, A. y Rodríguez, M. de la F. (2008). Estrés de rol, implicación con el trabajo y Burnout en soldados profesionales españoles. Revista Latinoamericana de Psicología, 40(2), 293-304.

Lorente, L., Salanova, M., Martinez, I. y Schaufeli, W. (2008). Extension of the Job Demands-Resources model in the prediction of burnout and engagement among teachers over time. Psicothema, 20(3), 354-360.

Manso, J. F. (2006). Confiabilidad y validez factorial del Maslach Burnout Inventory versión human services survey en una muestra de asistentes sociales chilenos. PsicologíaCientífica. com.

Martínez, A. (2010). El síndrome de Burnout: Evolución conceptual y estado actual de la cuestión. Vivat Academia, (112). Recuperado a partir de http://dialnet.unirioja.es/servlet/articulo?codigo $=3307970$

Maslach, C. y Jackson, S. E. (1984). Burnout in organizational settings. Applied Social Psychology Annual, 5, 133-153.

Maslach, C., Jackson, S. E. y Seisdedos, N. (1997). MBI: Inventario «Burnout» de Maslach : Síndrome del quemado por estrés laboral asistencial: Manual. Madrid: Tea.

Maslach, C., Leiter, M. P. y Schaufeli, W. (2008). Measuring burnout. En C. Cooper y S. Cartwright (Eds.), The Oxford handbook of organizational well-being (pp. 86-108).

Matteus, J. (2004). Programa de terapia de grupo cognitivo conductual para maestros de la escuela básica general del IPHE que presentan síndrome de burnout (Tesis para grado de maestría). Universidad de Panamá, Panamá.

Melamed, S., Shirom, A., Toker, S., Berliner, S. y Shapira, I. (2006). Burnout and risk of cardiovascular disease: Evidence, possible causal paths, and promising research directions. Psychological Bulletin, 132(3), 327-353. doi:10.1037/0033-2909.132.3.327

Miller, B. C. (2005). Characteristics of psychotherapists who are passionately committed to public mental health. Case Western Reserve University. Recuperado a partir de http://europepmc.org/abstract/ETH/3810

Olmedo, M., Santed, M. A., Jiménez, R. y Gómez, M. D. (2001). El síndrome de burnout: variables laborales, personales y psicopatológicas asociadas. Psiquis: Revista de Psiquiatría, Psicología y Psicosomática, 22(3), 11-23.

Ortega, C., Salas, R. y Correa, R. (2011). Aspectos epidemiológicos del síndrome de burnout en el personal sanitario. Hospital Aquilino Tejeira. Febrero-marzo 2011. Archivos de Medicina, 7(2:4). doi:10:3823/072 
Invest. pens. crit.

Vol. 2, No. 3, enero-abril 2014.

pp. $13-25$

Osca, A., González-Camino, G., Bardera, P. y Peiró, J. M. (2003). Estrés de rol y su influencia sobre el bienestar psíquico y físico en soldados profesionales. Psicothema, 15(1), 54-57.

Perczek, R., Carver, C. S., Price, A. A. y Pozo-Kaderman, C. (2000). Coping, mood, and aspects of personality in Spanish translation and evidence of convergence with English versions. Journal of Personality Assessment, 74(1), 63-87.

Pereda-Torales, L., Celedonio, M., Guillermo, F., Hoyos Vásquez, M. T. y Yánez Zamora, M. I. (2009). Síndrome de burnout en médicos y personal paramédico. Salud mental, 32(5), 399-404.

Quass, C. (2008). Diagnóstico de burnout y técnicas de afrontamiento al estrés en profesores universitarios de la quinta región de Chile. Psicoperspectivas. Individuo y Sociedad, 5(1), 65-75.

Rodes, M. (2006). Prevalencia del síndrome de burnout en los docentes de escuelas primarias oficiales y particulares en dos corregimientos del distrito de la Chorrera, 2004 (Tesis para grado de maestría). Universidad de Panamá, Panamá.

Rubio, J. C. (2003). Fuentes de estrés, síndrome de Burnout y actitudes disfuncionales en Orientadores de Instituto de Enseñanza Secundaria Obligatoria (Tesis doctoral). Universidad de Extremadura, España.

Schaufeli, W. B., Leiter, M. P. y Maslach, C. (2009). Burnout: 35 years of research and practice. Career Development International, 14(3), 204-220.

Shimizutani, M., Odagiri, Y., Ohya, Y., Shimomitsu, T., Kristensen, T. S., Maruta, T. y IImori, M. (2008). Relationship of nurse burnout with personality characteristics and coping behaviors. Industrial Health, 46(4), 326-335.

Shirom, A., Nirel, N. y Vinokur, A. D. (2006). Overload, autonomy, and burnout as predictors of physicians' quality of care. Journal of Occupational Health Psychology, 11(4), 328-342. doi:10.1037/1076-8998.11.4.328

Udipi, S., Veach, P. M., Kao, J. y LeRoy, B. S. (2008). The psychic costs of empathic engagement: Personal and demographic predictors of genetic counselor compassion fatigue. Journal of $G e$ netic Counseling, 17(5), 459-471. doi:10.1007/s10897-008-9162-3

Zur, O. (2007). Avoiding psychotherapists' burnout. New Therapist, 52, 22-26. 